\title{
POWER BANDWIDTH TRADE-OFF FOR SENSORY AND AD-HOC WIRELESS NETWORKS
}

\author{
Amir F. Dana and Babak Hassibi ${ }^{1}$ \\ California Institute of Technology \\ Dept. of Electrical Engineering, \\ Pasadena, CA, 91125, USA \\ \{dana, hassibi\}@systems.caltech.edu
}

\begin{abstract}
We look at the power bandwidth trade-off in random sensory and ad-hoc wireless networks with $n$ users and $r \leq \sqrt{n}$ simultaneous source/destination pairs. Under a specific protocol, we show that the minimum power required for maintaining an achievable scaling law of $R_{\text {sum }}=\Theta(f(n))$ for the sum-rate in the network, scales like $\Theta\left(\frac{f(n)}{\sqrt{n}}\right)$. The required bandwidth $B$ in this case is of order $\Theta\left(\frac{f(n)}{r}\right)$. It is also proved that the minimum achievable energy per information bit $\left(\frac{E_{b}}{N_{0}}\right)_{\min }$ for this protocol, scales as $\Theta\left(\frac{1}{\sqrt{n}}\right)$ and in this case the spectral efficiency is non-zero and is of constant order.
\end{abstract}

\section{EXTENDED ABSTRACT}

We consider power bandwidth trade-off in sensory and ad-hoc wireless networks. We assume that in both cases, there are $n$ users located randomly on a domain of fixed area $A$. At each time there are $r$ source/destination pairs in the network and all the other nodes act as relays (for the sensory case, $r=1$.). Based on the framework of [2], we look at the trade-off between the scaling behavior of the energy per bit (normalized to the noise spectral level) $\frac{E_{b}}{N_{0}}$ and the spectral efficiency $\mathcal{C}$. From [2], and the references therein, it is well known that for point to point communication over an AWGN or fading channel with average transmit power constraint, the minimum energy per bit $\left(\frac{E_{b}}{N_{0}}\right)_{\min }$ is achieved in the limit of $\mathcal{C} \rightarrow 0$. One important and interesting problem in the network setup is to determine the fundamental limits of $\frac{E_{b}}{N_{0}}$ and also the trade-off between $\frac{E_{b}}{N_{0}}$ and $\mathcal{C}$ (or equivalently power and bandwidth) in wireless networks. In this paper we look at this problem under a so-called "listen and transmit" protocol (see[1].).

We denote the transmit node and relay node powers by $p$ and $\sigma_{r}^{2}$ respectively. The channel model used in this paper is quite general and is the one used in [1]. Let $c_{x y}$ denote the channel between $x$ and $y$ in the domain $A$. We assume that, averaged over fading, the various channels are independent. For ad-hoc networks, we further assume that for any fixed points $y, z, t$ and $w$

$$
\begin{aligned}
& \mathrm{E}_{\{x\}} \mathrm{E} c_{x y}=0 \\
& \mathrm{E}_{\{x\}} \mathrm{E} c_{x y} c_{x z}=0 \\
& \mathrm{E}_{\{x\}} \mathrm{E} c_{x y} c_{x z} c_{x t} c_{x w}=0
\end{aligned}
$$

where $\mathrm{E}_{\{x\}}$ denotes the average over the location of node $x$ and the inner expectation is over the fading of the channels. The noise is also modeled as a zero mean white complex Gaussian random

\footnotetext{
${ }^{1}$ This work was supported in part by the National Science Foundation under grant no. CCR-0133818, by the office of Naval Research under grant no. N00014-02-1-0578, and by Caltech's Lee Center for Advanced Networking.
}

process with one-sided spectral level of $N_{0}$. Having said this, we state our results as the following theorems.

Theorem 1. Consider a sensory wireless network with $n$ relay nodes and one source/destination pair and the reception noise and channel model as described above. Then the minimum energy per information bit achievable with "listen and transmit" protocol and the corresponding spectral efficiency scale as $\Theta\left(\frac{1}{\sqrt{n}}\right)$ and $\Theta(1)$ respectively, i.e.,

$$
\left(\frac{E_{b}}{N_{0}}\right)_{\min } \stackrel{a . s .}{=} \Theta\left(\frac{1}{\sqrt{n}}\right), \quad \mathcal{C}=\Theta(1)
$$

Furthermore, any bandwidth and power allocation that satisfies $B \geq \frac{K}{\sqrt{n}}$ and $P_{\text {total }}=\frac{\alpha B N_{0}}{\sqrt{n}}$ for some positive constants $K$ and $\alpha$, achieves the scalings of (2).

A similar result can be stated for ad-hoc networks:

Theorem 2. Consider a wireless ad-hoc network with $n$ relay nodes, $r \leq \sqrt{n}$ source/destination pairs and the assumptions of (1). Then using the "listen and transmit protocol" the minimum energy per information bit $\left(\frac{E_{b}}{N_{0}}\right)_{\text {min }}$, over all the possible power and bandwidth allocations, and the corresponding spectral efficiency scale like

$$
\left(\frac{E_{b}}{N_{0}}\right)_{\min } \stackrel{a . s .}{=} \Theta\left(\frac{1}{\sqrt{n}}\right), \quad \mathcal{C}=\Theta(r) .
$$

Furthermore, these value are achieved for $\frac{r p}{N_{0} B}=\frac{n \sigma_{r}^{2}}{N_{0} B}=$ $\Theta\left(\frac{r}{\sqrt{n}}\right)$. Thus, as long as the source and relay node power per bandwidth is of this order, we can achieve $\left(\frac{E_{b}}{N_{0}}\right)_{\min }$ and spectral efficiency of order $\Theta(r)$.

Theorem 3. Consider a wireless ad-hoc network with $n$ relay nodes and $r \leq \sqrt{n}$ source/destination pairs and the assumptions of Theorem 2. Then for a given desired scaling law of $R_{\text {sum }}=\Theta(f(n))$ for the sum-rate in the network, the minimum required power for achieving that rate using "listen and transmit" protocol scales as

$$
P_{t}=\Theta\left(\frac{f(n)}{\sqrt{n}}\right)
$$

Also the required bandwidth for attaining that minimum power scaling law scales like $B=\Theta\left(\frac{f(n)}{r}\right)$.

\section{REFERENCES}

[1] A. F. Dana and B. Hassibi, "On the power efficiency of sensory and ad-hoc wireless networks," Submitted to IEEE Trans. Info. Theory, 2003.

[2] S. Verdú, "Spectral efficiency in the wideband regime," IEEE Trans. Info. Theory, vol. 48, pp. 1319-1343, 2002. 